\title{
Coherency between ground motions synthesized for analysis input of a large span structure
}

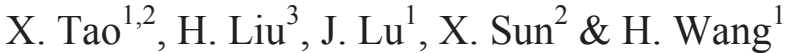 \\ ${ }^{1}$ Institute of Engineering Mechanics, China Earthquake Administration, China \\ ${ }^{2}$ School of Civil Engineering, Harbin Institute of Technology, China \\ ${ }^{3}$ State Key Laboratory of Bridge Structural Dynamics, \\ Chongqing Communication Research and Design Institute, China
}

\begin{abstract}
In order to fit the requirement of non-consistent input for seismic analysis of a large span structure with multi-supports, coherency of a synthesized ground motion field is validated in this paper. The result shows that the correlation between motions close to each other on rock site is strong, the mean values of coherencies are from 0.7 to 0.8 , and the maximum is up to 1.0 . The coherency decreases with the distance between the motion points. Correlation between low frequency motions close to each other on soil site is very strong, the mean of coherencies is larger than 0.8. The correlation of low frequency motions decreases also with the distance. The correlation between high frequency motions $(>5 \mathrm{~Hz})$ is weak, the coherencies corresponding to various distances are all less than 0.5 , and the mean is mostly less than 0.25 . The result is consistent with the conclusion from the statistics of the observed data on some dense arrays like SMART-1 during the past earthquakes.
\end{abstract}

Keywords: non-consistent input, ground motion, synthesis, coherency, multi-support.

\section{Introduction}

Seismic risk of a large span structure is generally evaluated from response analysis, since there are very few experiences of this kind of structure during past earthquakes. Non-consistent input for seismic analysis of a large span structure with multi-supports is emphasized in the last decades, from the spatial variation 
of ground motions observed at dense arrays (Berrah and Kausel [5]; Der Kiureghian and Neuenhofer [6]; Wang and Wang [26]). The simplest one is travelling wave, the waveforms are same, and the difference is just a time lag between the time histories. In order to describe the complicated nature of ground motion and reduce the risk from the input simplification, many improvements were suggested (Loh [13]; Harichandran and Vanmarke [7], Qu et al. [16]). The authors and their co-workers developed a set of approaches for synthesis for near field ground motion (Tao and Wang [21, 22]; Wang and Tao [25]; Zhang et al. [27]). It emphasized the predominant effect of source on the motion, took the nohomogeneous slip on the source rupture plane into account, the high frequency motion was randomly synthesized, the low frequency motion was calculated by means of a simplified numerical Green Function method, and the two were superposed in time domain after low and high pass filtered. For the validation of the approaches, the synthesized result was compared with some observed data during the past earthquakes. The distribution of peak values, response spectra of motions on the observation sites are quite similar, while the hanging wall effect and rupture directivity effect are described clearly (Liu et al. [12]; Sun [19]). This paper is going to validate if the approaches express the spatial structure of the real ground motion field, i.e. the spatial correlation between motions close to each other, in other words, the coherency in frequency domain. Obviously, this is very significant for the non-consistent input.

\section{Synthesis of ground motion field}

A hybrid source model is generated for the synthesis (Wang and TAO $[23,25]$ ), to describe the slip distribution of on the faulting plane of the earthquake with magnitude 6.5 , as shown in figure 1 .

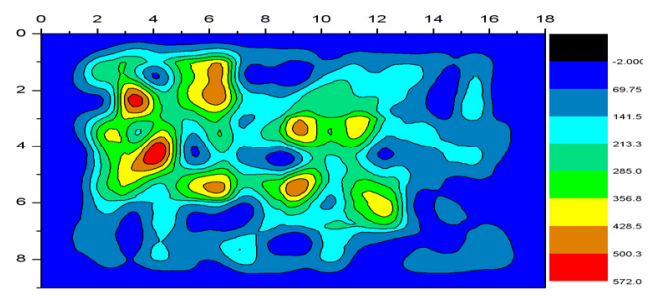

Figure 1: $\quad$ The adopted source model.

High frequency motion at ground surface is synthesized by a random approach (Atkinson and Silva [2]; Beresnev and Atkinson [3]). Fourier spectrum of ground motion from one sub-source is described in that approach as following (Boore [4]; Atkinson and Silva [2])

$$
F A\left(M_{0}, f, R\right)=S\left(M_{0}, f\right) \cdot G(R) \cdot D(R, f) \cdot A(f) \cdot P(f)
$$

where $\mathrm{S}(\mathrm{M} 0, \mathrm{f})$ is source spectrum, $\mathrm{G}(\mathrm{R})$ is geometry attenuation term, $\mathrm{D}(\mathrm{R}, \mathrm{f})$ is energy dissipation term, $\mathrm{A}(\mathrm{f})$ is near surface amplification factor, and $\mathrm{P}(\mathrm{f})$ if high frequency truncated function. A dynamic corner frequency is adopted in $\mathrm{S}(\mathrm{M} 0, \mathrm{f})$ 
so that the motion amplitude at near field and the received energy at far-field don't depend on the sub-source size (Motazedian and Atkinson [15]).

The motion at that ground point can be obtained by superposition of motions from all sub-sources with time lags according to the triggering time differences and the differences of distances from the sub-sources to the point, as follows (Tao and Wang [22]).

$$
a(t)=\sum_{i=1}^{N_{L}} \sum_{j=1}^{N_{W}} a_{i j}\left(t-\Delta t_{i j}\right)
$$

where $N_{L}, N_{W}$ are the number of the sub-sources along the strike and dip directions respectively, $a_{i j}(t)$ is the ground motion from the $i, j$ th sub-source, $\Delta t_{i j}$ is the corresponding time lag.

Low frequency ground motions are calculated by a simplified numerical Green function method where the regional media and 3D velocity structure of the crust in the volume containing the source and its surroundings are taken into account (Zhang et al. [27]). The whole region under consideration is divided into two parts, a deep homogenous zone with the source in it and a shallow inhomogeneous zone from the ground surface to bottom of the upper crust. The latter is divided further into finite element network. The displacement time history at each node on the bottom of the second part is from analytical solution of the first part. The ground motion at surface are calculated by a space-time decoupling explicit finite element analysis with a second-order local artificial transmitting boundary with displacement field input from the first step.

The wide band ground motion is superposed by corresponding high and low frequency motions in time domain at the every point filtered low and high pass respectively.

The site condition is taken into account by 1D equivalent linearization approach. In frequency domain, the ground motion at surface can be described as the multiple of the input at the bottom of soil layer and the transfer function of the soil layer.

$$
A(\omega)=R(\omega) \cdot U(\omega)
$$

where $A(\omega)$ is Fourier spectrum of motion at ground surface, $R(\omega)$ is the transfer function of soil layer, $U(\omega)$ is the spectrum of the input motion.

\section{Coherency coefficients}

Coherency function is considered as the best descriptor of the similarity and crossvariation between two ground motions. It is defined as following ( $\mathrm{Lu} \mathrm{[14])}$

$$
\gamma_{i j}(\omega)= \begin{cases}\frac{S_{i j}(\omega)}{\sqrt{S_{i i}(\omega) S_{j j}(\omega)}} & S_{i i}(\omega) S_{j j}(\omega) \neq 0 \\ 0 & S_{i i}(\omega) S_{j j}(\omega)=0\end{cases}
$$


where $S_{i j}(\omega)$ is cross-power spectrum of motions at the $i, j$ th points, $S_{i i}(\omega)$ and $S_{j j}(\omega)$ are the auto-power spectra of motions at the two points respectively. $\left|\gamma_{i j}(\omega)\right|$ is generally called as lag coherency, or coherency coefficient, $0 \leq\left|\gamma_{i j}(\omega)\right| \leq 1.0$. The bigger coherency coefficient, the stronger relativity is between the motions at the two points. When the value of the coefficient is 1.0, the motions are the same completely.

Let $a_{i}(t)$ and $a_{j}(t)$ are the acceleration time histories at the $i, j$ th points, the coherency coefficient is calculated by the following process:

(1) To calculate the Fourier spectra $A_{i}(\omega)$ and $A_{j}(\omega)$ of $a_{i}(t)$ and $a_{j}(t)$ respectively by Fast Fourier Transfer (FFT).

(2) To calculate the corresponding cross power spectrum and auto-power spectra by

$$
\begin{gathered}
S_{i i}(\omega)=A_{i}^{*}(\omega) A_{i}(\omega) \\
S_{j j}(\omega)=A_{j}^{*}(\omega) A_{j}(\omega) \\
S_{i j}(\omega)=A_{i}^{*}(\omega) A_{j}(\omega)
\end{gathered}
$$

where $A_{i}^{*}(\omega)$ and $A_{j}^{*}(\omega)$ represent the conjugation of $A_{i}(\omega)$ and $A_{j}(\omega)$, respectively.

(3) To smooth the spectra in frequency domain by

$$
\bar{S}(\omega)=\int_{-\infty}^{+\infty} S(\Omega) w(\omega-\Omega) d \Omega
$$

where $\bar{S}(\omega)$ represents $S_{i i}(\omega), S_{j j}(\omega)$ or $S_{i j}(\omega)$ respectively, $w(\omega)$ is spectrum window that is to restrain the leak in FFT. The Parzzen window is adopted here

$$
w(\omega)=\frac{3}{4} u\left(\frac{\sin \frac{u \omega}{4}}{\frac{u \omega}{4}}\right)^{4}
$$

where $u$ is a parameter of band width of the spectrum window, it can be determined from the equivalent band width $\mathrm{b}$ as follows

$$
u=\frac{280}{151 b}
$$

The bigger band width $b$ is the smoother is the spectrum.

(4) To calculate the coherency by

$$
\left|\gamma_{i j}(\omega)\right|=\frac{\left|\bar{S}_{i j}(\omega)\right|}{\sqrt{\bar{S}_{i i}(\omega) \bar{S}_{j j}(\omega)}}
$$




\section{Coherency between ground motions in the synthesized field}

\subsection{Coherency coefficient from ground motion field on rock site}

A line is selected from the area of the synthesized motion field motioned in section 2 of this paper, and then a set of points with 200 meter intervals on the line are chosen. The coherencies are calculated for motions at point pairs with various distances, by means of the procedure introduced in section 3 . Figure 2 shows two examples of coherencies between motions at points with distance $200 \mathrm{~m}$ to each other on rock site.

From the figure, one can see that the coherency coefficient between motions at points with 200 meter distance on rock surface varies with frequency with a

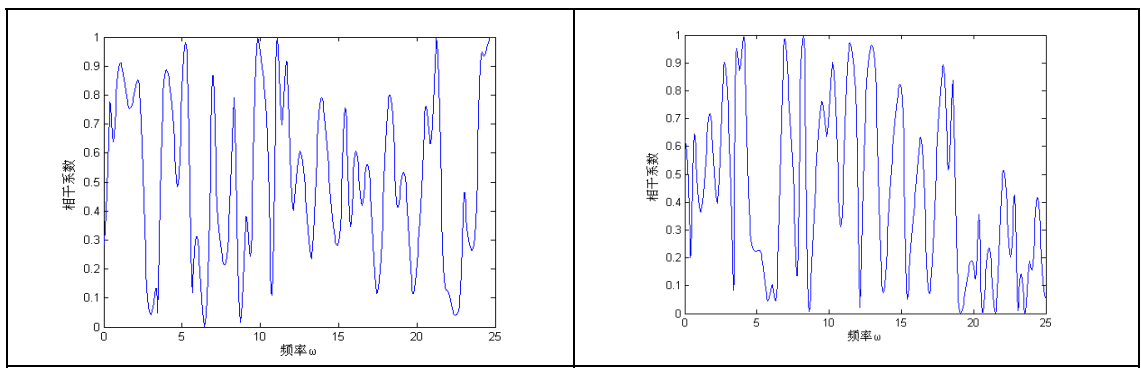

Figure 2: Two examples of coherencies between motions at points with distance $200 \mathrm{~m}$ on rock site.

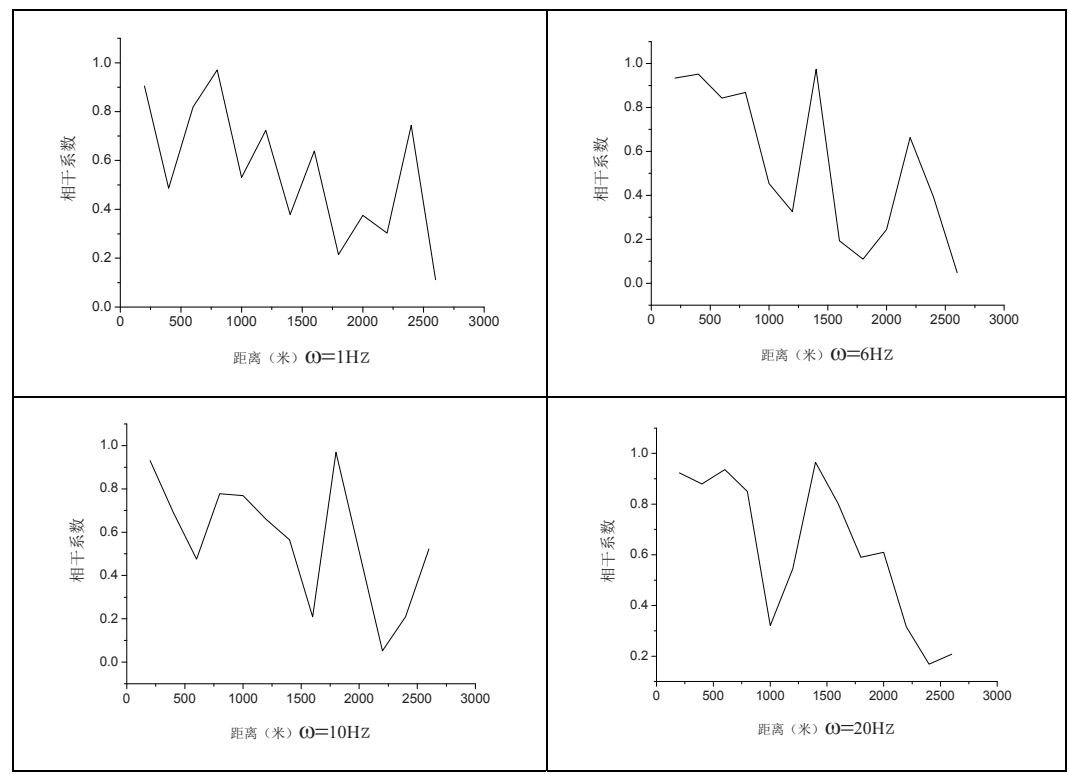

Figure 3: Variation of the coherency with distance at 4 frequencies on rock site. 
fluctuation and without a certain trend, and the mean value is from 0.7 to 0.8 , maximum is up to 1.0. It means the spatial correlation is strong.

Figure 3 shows the coherency coefficients between the points with distance from 200 to 2800 meters at 4 frequencies 1.0, 6.0, 10.0 and $20.0 \mathrm{~Hz}$. One can see from the figure that the coefficient decreases while the distance increases. The statistic result shows that coherencies of the motions at point pairs with distance no larger than 400 meters are almost more than 0.8 and those correlations are small for distance thousand meters.

\subsection{Coherency coefficient from ground motion field on soil site}

Figure 4 shows two examples of coherencies between motions at points with distance $200 \mathrm{~m}$ to each other on soil site, for North-South, East-West and UpDown directions respectively, from up to down.

From the figure one can see that the coherency is quite strong in the range of frequency less than $5 \mathrm{~Hz}$, the coefficient values are more than 0.8 in average. For the range of frequency larger than $5 \mathrm{~Hz}$, coherency decreases quickly, the mean of coefficient is from 0.2 to 0.3 . It means the correlation in that rage is weak.

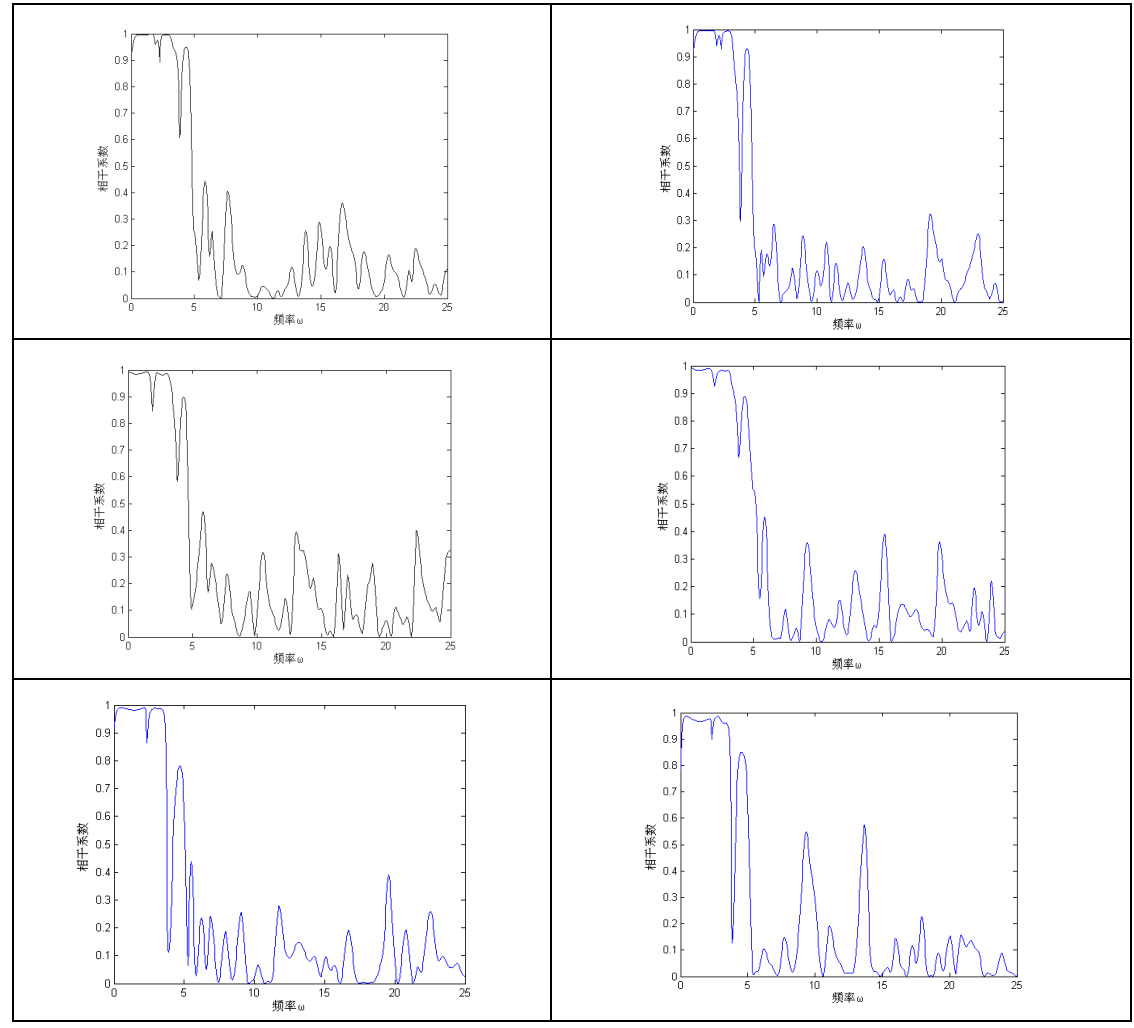

Figure 4: Two examples of coherencies between motions at points with distance $200 \mathrm{~m}$ on soil site. 


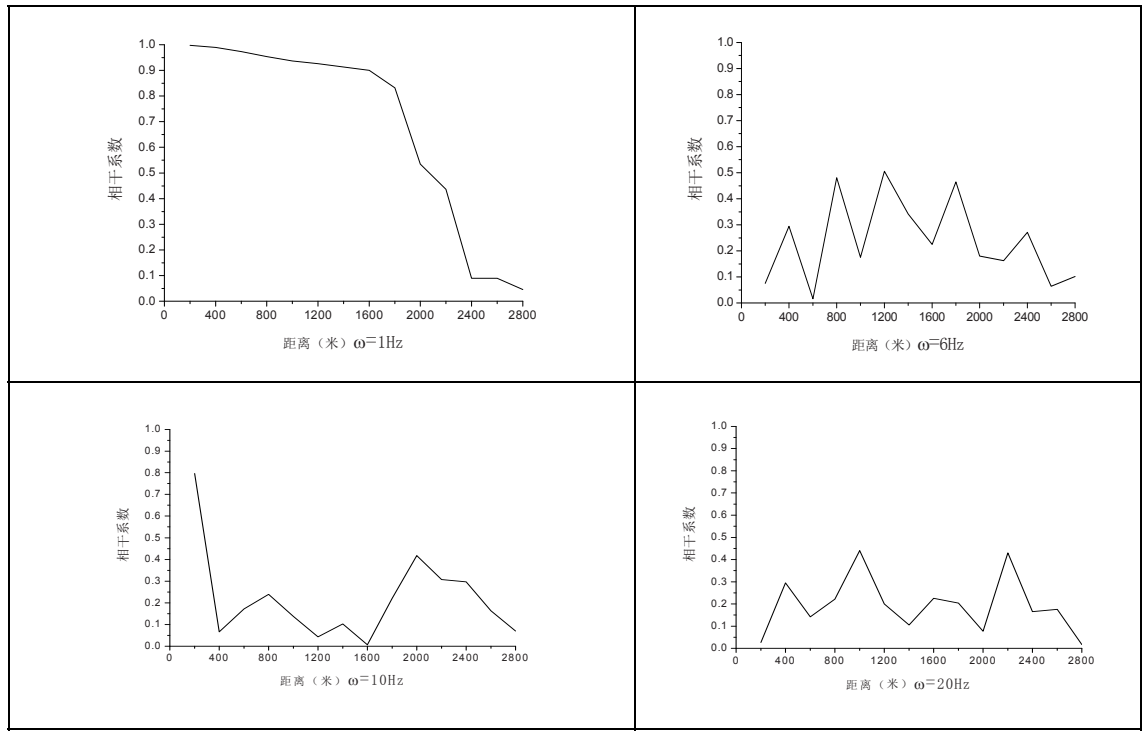

Figure 5: Variation of the coherency with distance at 4 frequencies on soil site (NS).

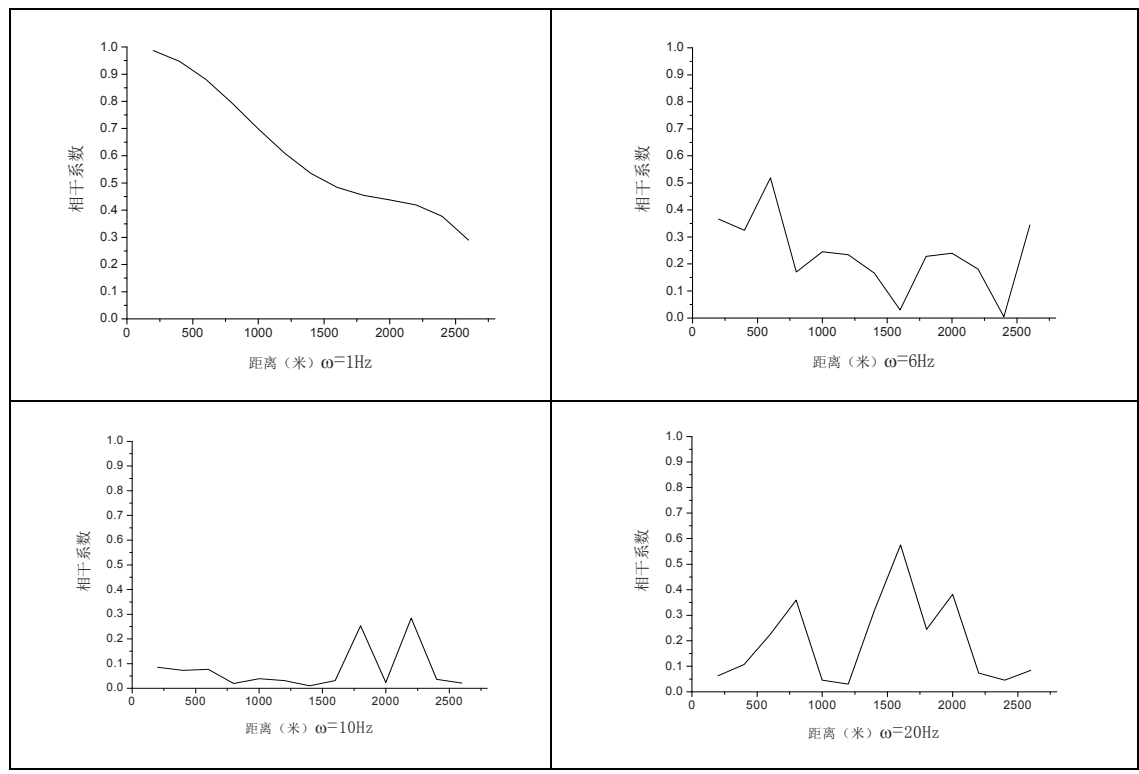

Figure 6: Variation of the coherency with distance at 4 frequencies on soil site $(\mathrm{EW})$.

Figure 5, figure 6 and figure 7 show the coherency coefficients between the points with distance from 200 to 2800 meters at the 4 frequencies, for North- 
South, East-West and Up-Down directions respectively, from up to down. One can see from those that the correlation is large for low frequency $(1.0 \mathrm{~Hz}$ and less) motions with distance 400 meters and less, the coefficients are all more than 0.8 , and it decreases with frequency quickly. Similarly, it also decreases with distance. Coherency of high frequency motions is weak, the coefficients are all less than 0.5 , and the mean values are almost less than 0.25 .

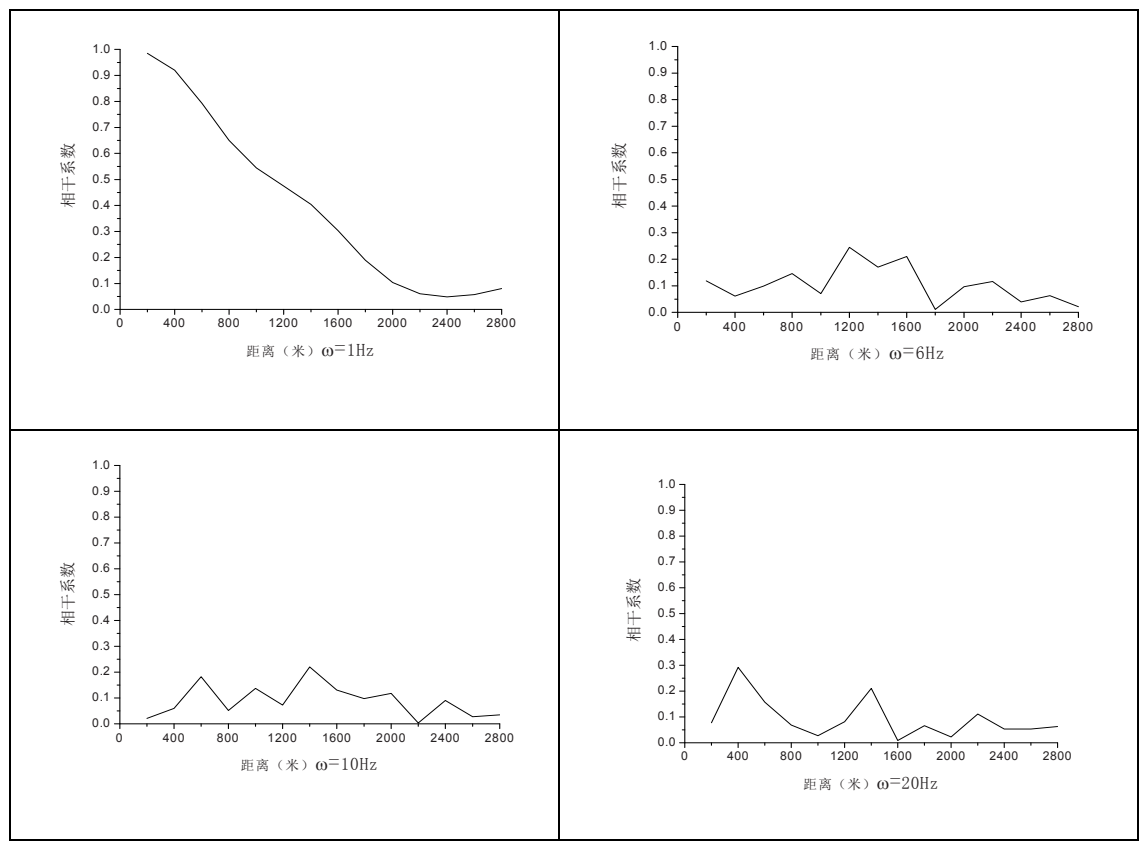

Figure 7: Variation of the coherency with distance at 4 frequencies on soil site (UD).

\section{Conclusions}

Spatial structure of a synthesized ground motion field is validated by means of the coherency coefficients between motion pairs with various distances. The result shows that the correlation between motions with distance 200 meters on rock site is strong, the mean values of coherencies are from 0.7 to 0.8 , and the maximum is up to 1.0. There is a trend that coherency decreases with the distance, the coefficient between motions with distance 400 meters and less are almost more than 0.8 and it gets small for thousand meter distance. Correlation between low frequency motions with distance 200 meters on soil site is very strong, the mean of coherencies is larger than 0.8. The correlation of low frequency motions decreases also with the distance. The correlation between high frequency motions $(>5 \mathrm{~Hz})$ is weak, the coherencies corresponding to various distances are all less than 0.5 , and the mean is mostly less than 0.25 . 
The result of this paper is consistent with the conclusion from the statistics of the observed data on some dense arrays like SMART-1 during the past earthquakes, the spatial correlation of ground motions at two surface points depends on the distance and frequency components of the motions, decreases while distance increases and/or frequency is getting high (Jin and Liao [9]).

\section{Acknowledgements}

This work was supported by National Natural Science Foundation of China under Contract No. 50778058 and 90715038, and the Fund of Key Laboratory of Bridge Earthquake Resistance Technology, Ministry of Communications, P. R. C. under Contract No. 200802.

\section{References}

[1] Aki. K, An overview and issues: review of simulation procedures, Strong ground motion simulation and earthquake engineering applications, EERI, 1985

[2] Atkinson G. and W. Silva. Stochastic modeling of California ground motions. Bulletin of the Seismological Society of America, 90(2): 255-274. 2000

[3] Beresnev, I. and G. Atkinson. Source parameters of earthquakes in eastern and western North America based on finite-fault modeling. Bulletin of the Seismological Society of America, 92:695-710. 2002

[4] Boore, D. M. (1983). Stochastic simulation of high-frequency ground motions based on seismological models of the radiated spectra. Bulletin of the Seismological Society of America 73(6): 1865-1894

[5] Berrah M., Kausel E. Response spectrum analysis of structures subjected to spatially varying motions. EESD, 21(6):461-470. 1992

[6] Der Kiureghian A., Neuenhofer A. Response spectrum method for multi support seismic excitations. EESD, 21 (8):713-740. 1992

[7] Harichandran R., E. Vanmarke. Stochastic Variation of Earthquake Ground in Space and Time. ASCE. J. EM. 112(2):154-174. 1986

[8] Irikura, K., Prediction of strong motions from future earthquakes caused by active faults-case of the Osaka basin, Proc. of 12WCEE, Auckland, 2000

[9] Jin X. and Z. Liao. Physical simulation of random field of ground motion. Earthquake engineering and engineering vibration, 14(3):11-19. 1994

[10] Kakehi, Y. and K. Irikura, Estimation of high-frequency wave radiation areas on the fault plane by the envelope inversion of acceleration seismograms, Geophys. J. Int., 125, 892-900. 1996

[11] Liu Haiming, Tao Xiaxin, Sun Xiaodan, and Li Ping. Forecast of strong ground motion field near the fault for earthquake disaster reduction in urban areas. Proc. of the 7th International Conference on Earthquake Resistant Engineering Structures, Cyprus, 2009

[12] Liu H. M., X. X. Tao, X. D. Sun, P. Li, Forecasting of the strong ground motion field in Lanzhou city and its vicinity caused by an earthquake with magnitude 6.5 on the western segment of Maxianshan-beiyuan fault, Northwestern Seismological Journal, 30(3): 227-231 (in Chinese). 2008 
[13] Loh C. H., Analysis of the spatial variation of seismic waves and ground movements from SMART-1 Array data, EESD, 13(5): 561-581, 1985

[14] Lu Jianbo. Spatial correlation of synthesized ground motion field. Dissertation for Master Degree, Institute of Engineering Mechanics, 2008 (in Chinese)

[15] Motazedian D. and G. M. Atkinson. Stochastic finite-fault modeling based on a dynamic corner frequency. Bulletin of the Seismological Society of America, 95(3): 995-1010. 2005

[16] Qu Tiejun, Wang Junjie, Wang Qianxin. A practical model for the power spectrum of spatial variant ground motion. ACTA Seismologica Sinica, 18(1):55-62. 1996

[17] Somerville, P.G., R.W. Graves, Strong ground motions of the Kobe, Japan earthquake of Jan. 17, 1995, and development of a model of forward rupture directivity effects applicable in California. Proc. of the Western Regional Technica; Seminar on earthquake engineering for dams, Association of State Dam Safety Officials, Sacramento, 1996

[18] Somerville P., Seismic hazard evaluation, Proc. 12WCEE, Auckland, 2000

[19] Sun Xiaodan, Some issues on estimation of strong ground motion field. Dissertation for Doctor Degree, Harbin Institute of Technology (in Chinese), 2010

[20] Tao Xiaxin, Sun Xiaodan, Liu Haiming and Li Ping. Some issues on forecast of strong ground motion field. Proc. of the 7th International Conference on Earthquake Resistant Engineering Structures, Cyprus, 2009

[21] Tao Xiaxin and Wang Haiyun, A random source model for near filed strong ground motion prediction, Proc. of 13WCEE, Vancouver. Paper No.1945. 2004

[22] Tao Xiaxin, Wang Guoxin. Rupture directivity and hanging wall effect in near field strong ground motion simulation, ACTA Seismologica Sinica, 16(2): 205-212. 2003

[23] Wang Haiyun and Tao Xiaxin, Relationships between moment magnitude and fault parameters: theoretical and semi-empirical relations, Earthquake Engineering and Engineering Vibration, 2(2):201-211. 2003

[24] Wang Haiyun, Tao Xiaxin, Li Jie, Global source parameters of finite fault model for strong ground motion predictions, Proc. of 13WCEE, Vancouver. Paper No.2743. 2004

[25] Wang H. Y. and X. X. Tao, Charactering a shallow earthquake asperity model for predicting near field strong ground motion, Journal of Harbin Institute of Technology, 37(11): 1533-1539 (in Chinese). 2005

[26] Wang Junjie, Wang Qianxin. Response of large spam arc bridge under ground motion with spatial variation. Journal of Vibration Engineering, 8(2):119-126. 1995

[27] Zhang D. L., X. X. Tao, Z. H. Zhou, Study on Finite Fault Model for Calculation-Considering Slip-Time Function of Heterogeneous Displacement Distribution in Temporal and Spatial Domain. Northwestern Seismological Journal, 27(3):193-198 (in Chinese). 2005 